\title{
PREDICTIVE MODELS FOR UNDERGRADUATE STUDENTS PERFORMANCE ON THE DATABASES COURSE
}

\author{
Adina DIACONU \\ Al. I. Cuza University, Faculty of Economics and Business Administration, Iasi, Romania \\ adinadcn@gmail.com \\ Ionuţ HRUBARU \\ Al. I. Cuza University, Faculty of Economics and Business Administration, Iasi, Romania \\ ionuţ.hrubaru@feaa.uaic.ro
}

\begin{abstract}
Predictive modeling has been a long-debated subject in the Big Data era. In this paper, we were concerned with developing predictive models for undergraduate students' performance in the Databases course, at the Faculty of Economics and Business Administration, Alexandru Ioan Cuza University, Iaşi using CART and Random Forest algorithms. Also, the accuracy of the models was investigated, proving that the Random Forest algorithm is more suitable for the test data which was collected.
\end{abstract}

Keywords: Predictive modelling, CART, Random Forest, students' performance

JEL classification: C53, C61

DOI: $10.12948 / \mathrm{ie} 2019.03 .06$

\section{Introduction}

We are in an era often called the information age. With the enormous amount of data stored in files or databases, often referred as Big Data, it is necessary to develop powerful tools of analyzing and interpreting it in order to support the decision-making process. In this context, data mining, also known as Knowledge Discovery in Databases (KDD), refers to the selection and analysis of clustered data to find and confirm previously unknown relationships that can produce positive and verifiable results by implementing predictive models when new data appears [1].

There are three main steps in the automatic learning process. In the first phase, preprocessing, data is collected, reformatted and transformed. Then, with the necessary data set, in the second phase, models can be built. The last phase validates and measures how well the models work. This way, the results are interpreted, while being a decision-making phase at the same time. Therefore, one can try to understand how well our models work with data that they have not seen before. Metrics such as accuracy, precision, and sensitivity, are used. If the results are not satisfactory, we can resume any previous phase. These three steps are the basic stages of machine learning models. In practice, these steps are repeated. Each iteration can provide new information about our data and hypotheses and contribute to improving models.

\section{Brief Literature Review}

A great challenge faced by a data scientist is collecting the data needed for the analysis. Starting from this point, many questions arise such as: "Is this data good?". When it comes to how good data is, the following questions need to be answered: Is our data relevant? Is it connected to the problem we are trying to solve? On the other hand, do we miss much data? Is the data correct? Do we have enough data to answer a specific question? Therefore, the relevant, correlated, accurate and sufficient data are the four pillars of how good our data is [2]. 
An algorithm in data mining or in automatic learning is a set of heuristics and calculations that create a data model. However, to create it, the algorithm first looks at the data and searches for specific types of patterns or trends. A number of advantages and disadvantages characterize each algorithm. Some may be faster, some slower. Some offer accuracy, others do not. And the list goes on. There are many data processing algorithms, but we will only focus on those who will be part of the methodology of this paper.

S. Serengil [3] states that an algorithm is transparent if its decisions can easily be read and understood by people. Among the most popular algorithms that lead to transparent decisions are decision trees. The most well known and often used algorithms that implement decision trees are CART (Classification and Regression Trees). Usually, this algorithm is called a decision tree, but within the $\mathrm{R}$ platforms, reference is made to the more recent term CART.

Classification and regression trees are characterized by a set of rules to be applied to an unclassified data set to estimate which records will return a given output. To get this result, it segments the data set, creating binary trees. The CART algorithm consists of a series of questions, and their answers determine the next question. The representation of the CART is a binary tree, where the ends are terminals, when there are no questions. Each subset of its component represents a response to a classification question. The tree leaves are partitions or segmentations of the data set according to the classification, and the nodes present statistical information. Therefore, the main idea around which to build decision trees is to use a criterion division to determine the predictive factor and the location of the first point of decision tree, and to perform a search of predictors to build trees until there is no data to process [4]. The CART algorithm is also the basis of important algorithms such as random forest or boosting. Decision tree and their extension, Random Forest, are robust and easy to interpret algorithms for automated learning, classification and regression activities. One of the greatest advantages of using decision trees and random forests is the ease with which we can see what characteristics or variables contribute to classification or regression as well as their relative importance depending on their position in depth of the tree.

One key element to notice is that each automated learning algorithm should be viewed as a potential tool. Doug R. [5] recommends experimenting to find the best algorithm or one can choose to work with several different tools to improve the accuracy of the results. One thing to remember is that different algorithms can give different results, but generally, they should be as close as possible.

\section{Method, tools}

For this paper, we wanted to develop predictive models for undergraduate students' performance on the databases course using CART and Random Forests algorithms. This analysis, as well as these predictive models, are based on the background of students from the Faculty of Economics and Business Administration, Alexandru Ioan Cuza University, Iaşi (the grade obtained at the baccalaureate exam, the profile of the graduated class) and the grades obtained at other disciplines (Mathematics applied in economy, finance, etc.).

Data collection is a very important stage as mentioned in previous sections and requires considerable time and effort. Having the necessary data, we can move on to the next step, building the model. Next, we propose to define models for undergraduate students' performance on the databases course based on student history (baccalaureate grade, graduate class profile) and grades from other disciplines.

This model was based on 2195 students and 152 variables. The main variables were:

- Admission_year,

- Admission_gpa,

- Bacc_gpa - baccalaureate grade, 
- Hs_concentration_ro -graduate class profile,

- Hs_city,

- Finance,

- Money_and_credit,

- End_user_computing and other disciplines.

Data analysis was performed with R (R Core Team, 2017) and RStudio platforms. The main R libraries used were: tidyverse, rpart (for Regression Tree) randomForest, mice (needed to handle missing values).

\section{Results and discussions}

We initially submitted the data set to the CART algorithm. As expected, the first tree contained many nodes, more exactly 10839, making it almost impossible to decipher. As a result, we have resorted to tree pruning to increase accuracy for inconsistent data. Such a tree has been built by specifying the complexity parameter, but it cannot be easily read in this case, consisting of 109 nodes. We notice that the number of nodes dropped significantly, but not enough. So, we continued this process of reducing the tree, reaching the expected result. In Figure 1 is the 47node regression tree. At this point, it is much easier to interpret the results.

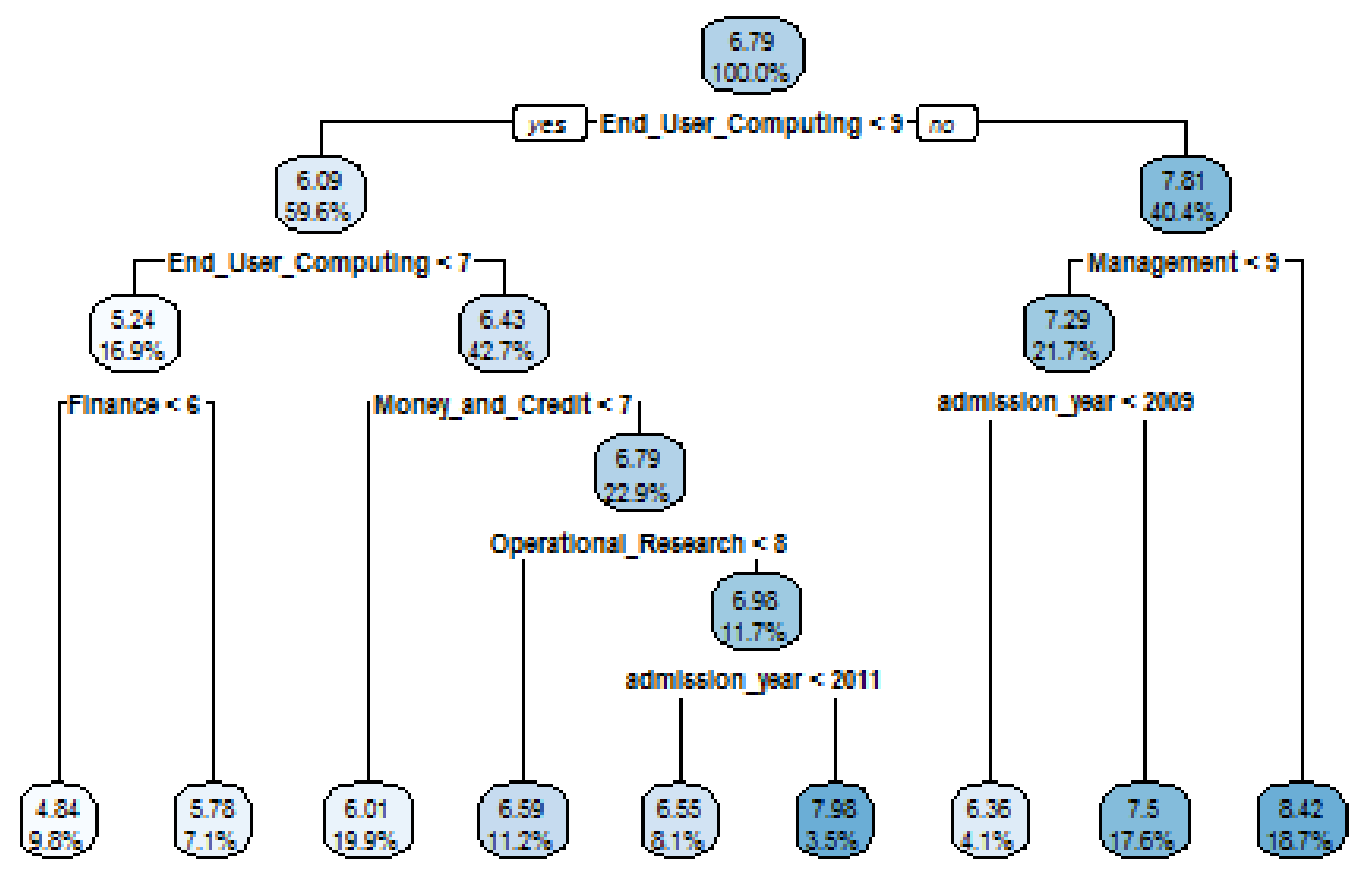

Figure 1. Regression tree

According to the tree, we can conclude that the average from the Databases can be predicted according to the following parameters: the grade obtained in the End User Computing, Management, Money and Credit, Finance, Operational Research disciplines and the admission year.

Therefore, for a student who obtained an 8 grade at End User Computing, but a 6 grade at Money and Credit, it is likely that he will get a 6 grade at the Databases disciplines. We can 
take another example: a student who has a 10 grade at End User Computing and over 9 at Management, can get 8.42 on Databases.

Figure 2 depicts the graphical representation of the variables which can be used in determining this model. Therefore, the most important predictor in this model is the note from the discipline Money and Credit, followed by the grade from Finance, End User Computing and Management.

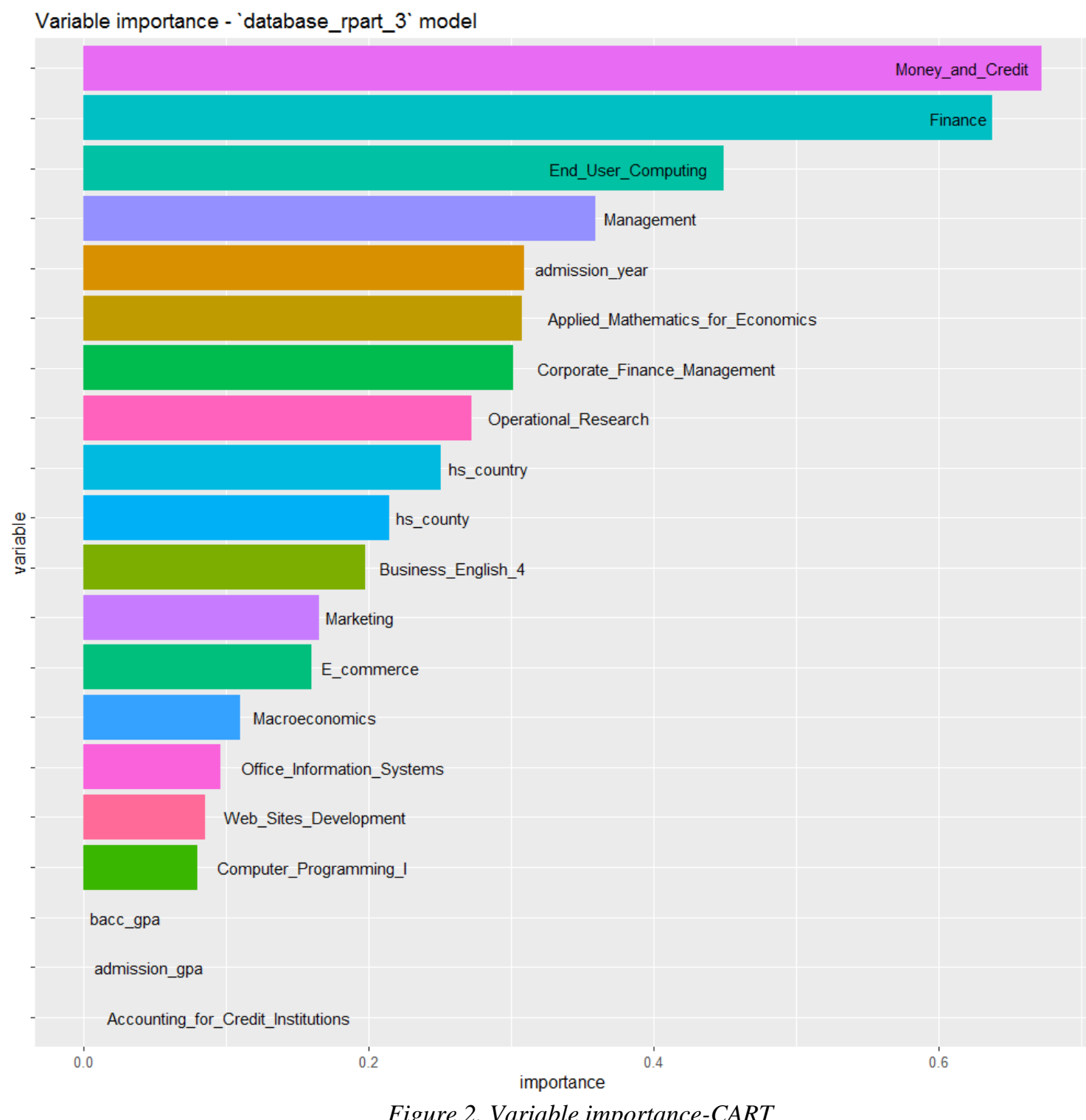

Next, we submitted the same data set to another algorithm: Random forests. Unlike the importance of the variables obtained in the CART-based predictive model, in this model, depicted in Figure 3, the most important predictor is the grade obtained at End User Computing, followed by the grades on Finance, Money and Credit and Management disciplines. So, according to Random forests, the order of the predictors is different from the one previously obtained. 


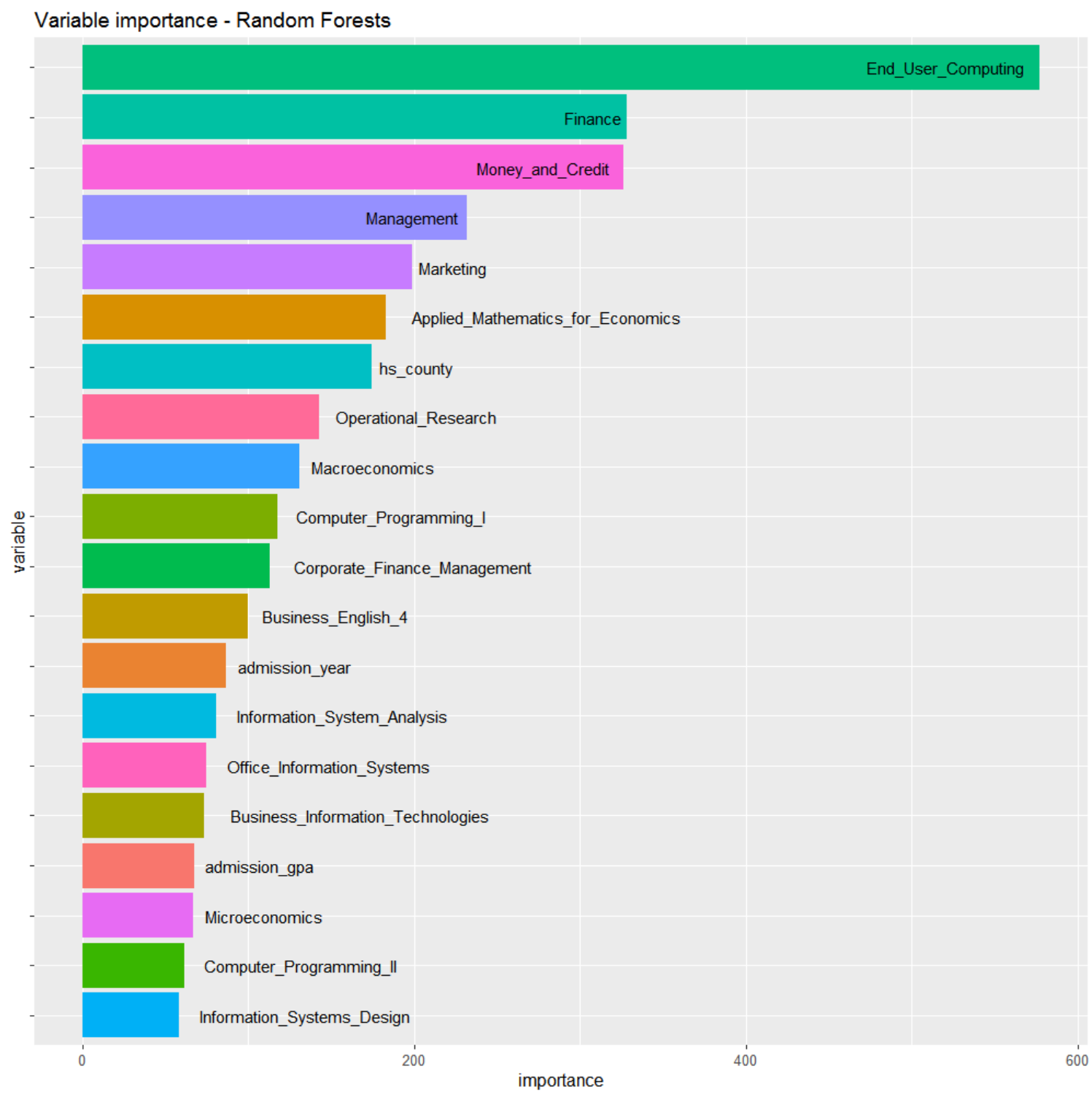

Figure 3. Variable importance-Random Forests

\section{Conclusions and further research}

To compare the two models, we have to take into account the values of two parameters: root mean square error and R-square. The root mean square error (RMSE) value is a commonly used measure of the differences between the values (sample and population values) predicted by a model and the values actually observed. In other words, it is a measure of the expected error of the estimator. RMSE for training and test sets should be very similar if we built a good model. If the RMSE for the test set is much higher than the training set, we may not have chosen the data well. This means that these two measures are used to estimate how well they were able to predict the test results. Therefore, for the 47-node model, we obtained an RMSE value equal to 1.283736 , and a RMSE value equal to 1.089029 was obtained through Random forests. As we mentioned earlier, the lower this value, the better the model is. By comparing the other parameter, R square, we come to the same conclusion, since a higher value indicates a better choice of the model. In our case, the $\mathrm{R}$ square value using Random forests was 0.5688894. This result being greater than $\mathrm{R}$ square for the CART model, 0.3954403, we can state that the best model for this data set is the one generated using Random forests. 
www.conferenceie.ase.ro

Although we have stopped on these two algorithms, in the future we can also try a multitude of other different algorithms and then look at the results more closely. As we mentioned earlier, it is advisable to experiment with the theoretical part in order to find the best algorithm. In this way, we can improve the accuracy of the results. Different algorithms can yield different results, but generally, they should be as close as possible, as it has been shown in our case.

\section{References}

[1] J. Han, M. Kamber and J. Pei, Data Mining: Concepts and Technique. Third Edition. SUA: Morgan Kaufmann, 2011, p.24.

[2] S. Malik. How good is your data? [Online]. Available: https://www.linkedin.com/learning/azure-machine-learning-development-1-basicconcepts/how-good-is-your-data?u=2057564, August 21, 2018. [Accessed Mar. 2, 2019].

[3] S. I. Serengil. A Step by Step CART Decision Tree Example. [Online]. Available: https://sefiks.com/2018/08/27/a-step-by-step-cart-decision-tree-example, August 27, 2018, [Accessed Mar. 2, 2019].

[4] A. Nepomnjashiy. Data Mining Algorithms: Microsoft SQL Server 2000 vs. "Yukon" SQL Server. [Online].

Available: https://www.databasejournal.com/features/mssql/article.php/3305411/Data-MiningAlgorithms-Microsoft-SQL-Server-2000-vs-Yukon-SQL-Server.htm, February 3, 2004, [Accessed Mar. 2, 2019].

[5] D. Rose. Select the best algorithm. [Online]. Available: https://www.linkedin.com/learning/artificial-intelligence-foundations-machine-

learning/select-the-best-algorithm?u=2057564, March 29, 2018, [Accessed Mar. 2, 2019]. 